\title{
Area Spectral Efficiency of Soft-Decision Space-Time-Frequency Shift Keying Aided Slow Frequency Hopping Multiple Access
}

\author{
Hoang Anh $\mathrm{Ngo}^{\dagger}$ and Lajos Hanzo ${ }^{\dagger}$ \\ $\dagger$ School of Electronics \& Computer Science, University of Southampton \\ Email: ${ }^{\dagger}\{$ han08r, lh\}@ecs.soton.ac.uk; http://www-mobile.ecs.soton.ac.uk
}

\begin{abstract}
Slow Frequency Hopping Multiple Access (SFHMA) is capable of providing inherent frequency diversity and of beneficially randomising the effects of cochannel interference. It may also be advantageously combined with our novel Space-Time-Shift Keying (STFSK) scheme. The proposed system's area spectral efficiency is investigated in various cellular frequency reuse structures. Furthermore, it is compared to both classic Gaussian Minimum Shift Keying (GMSK) aided SFHMA as well as to GMSK assisted Time Division/ Frequency Division Multiple Access (TD/FDMA). The more sophisticated third-generation Wideband Code Division Multiple Access (WCDMA) and the fourthgeneration Long Term Evolution (LTE) systems were also included in our comparisons. We demonstrate that the area spectral efficiency of the STFSK aided SFHMA system is higher than those of the GMSK aided SFHMA and TD/FDMA systems as well as that of the WCDMA, but it is only $60 \%$ that of the LTE system.
\end{abstract}

\section{INTRODUCTION}

Slow Frequency Hopping Multiple Access (SFHMA) [1] not only provides inherent frequency diversity but also advantageously randomises the effects of cochannel interference. Furthermore, it is capable of avoiding the problem of prolonged fades typically experienced by stationary or slow moving Mobile Stations (MS) since hopping to another independently faded frequency might curtail fading. The classic SFH900 mobile system [2], [3] which was based on "mixed" Slow Frequency Hopping (SFH) combined with time division demonstrated the benefits of SFHMA.

Owing to its potential to increase the attainable system capacity without requiring additional bandwidth, Multiple-Input-MultipleOutput (MIMO) techniques have been extensively investigated. The family of MIMO systems typically employs Spatial Division Multiplexing (SDM), such as the BLAST schemes of [4], [5] for increasing the transmission rate or Space-Time Codes (STC) [6], [7] for maximizing the attainable diversity order. More recently, Spatial Modulation [8] and Space Shift Keying [9] were proposed for avoiding any inter-antenna interference and inter-antenna synchronization. As a further advance, Space-Time-Frequency Shift Keying (STFSK) [10] was designed in order to achieve a beneficial diversity gain, which may be gleaned from three different domains, namely the space-, time- and frequency-domain.

All the above-mentioned techniques play an important role in the overall system design, but they have typically been investigated independently. Therefore, in this paper we develop the philosophy of STFSK into a multi-user, multi-cell SFHMA system in order to investigate the inter-play of these techniques on the performance of

The research leading to these results has received funding from the European Union's Seventh Framework Programme ([FP7/2007-2013]) under grant agreement no [214625]. The financial support of RC-UK under the auspices of the UK-India Advanced Technology Centre as well as that of the China-UK Science Bridge in 4G Wireless Communications is also gratefully acknowledged. the holistically optimized system. The novelty and rational of our proposed scheme is summarized as follows:

1) First, we intrinsically amalgamate the STFSK with SFHMA and then investigate the achievable Area Spectral Efficiency (ASE) of a realistic multi-user, multi-cell wireless environment under different cellular frequency reuse structures.

2) The study in Chapter 7 of [11] showed that the SFHMA system is superior in comparison to the Time Division/Frequency Division Multiple Access (TD/FDMA) benchmarker for a low-complexity $16 \mathrm{kbit} / \mathrm{s}$ subband speech codec combined with Reed-Solomon coding. By contrast, in this paper we demonstrate that the SFHMA system becomes inferior to the TD/FDMA system when state-of-the-art components, such as the Advanced Multi-rate (AMR) speech codec and the convolutional coding are employed.

3) Finally, we demonstrate that by intrinsically amalgamating low-complexity STFSK and SFHMA, we attained an improved ASE, compared to those of TD/FDMA and WCDMA. However, this ASE remains lower than that of the more complex LTE system, when the same convolutional channel code and the same system bandwidth are employed.

The outline of this paper is as follows. The SFHMA's frequency reuse structures are presented in Section II. In Section III, we investigate the spectral efficiency of the STFSK aided SFHMA. Finally, our concluding remarks are offered in Section IV.

\section{Slow Frequency Hopping Multiple Access (SFHMA)}

The SFHMA technique employs SFH relying on unique, userspecific pseudo-random hopping sequences for supporting multiple users. The SFH family may be divided into three categories, namely the orthogonal, random and mixed protocols [11]. As a benefit of providing an increased freedom in system design and of its improved spectral efficiency [2], [3], the mixed protocol will be considered in the following sections.

The basic form of the reuse structure has three basic frequencysets, also often referred to as 'colours' [2], [3]. Each colour is represented by one of the letters A, B, C and each contains a set of $N_{c}$ frequencies. For a given reference cell associated with colour A, all other cells marked A are full-reuse cells, relying on the same set of frequencies. For the full-reuse structure, an active user in the reference cell will experience interference from an active user in the reuse cell, during one of each sequence of $N_{c}$ hops, when they happen to use the same frequency. Each additional active user from the same or other reuse cell will impose the same frequency collision probability, but at a different frequency in the reference user's sequence. Therefore, the so-called 'frequency collision rate' is defined as the proportion of the hopping sequence, when a 'hit' by an identical-frequency active user is encountered. For a full reuse cluster size $C_{c}$, the frequency collision rate equals to $C_{c} / 3 N_{c}$. 
By contrast, in a fractional reuse structure, each colour is divided into $M_{c}$ overlapping sub-sets of $L_{c}$ groups of frequencies, which are referred as 'shades' of that colour, or 'pseduo-colour' [2]. Hence, the frequency-reuse cluster contains $3 M_{c}$ cells divided into $M_{c}$ subclusters of size 3 , each using a different shade of 3 colours. This is known as a $3 M_{c} / L_{c}$ fractional structure [2], where $L_{c} / M_{c}$ is the fraction of the $N_{c}$ frequencies making up a colour, which is constituted by the set of shades. Fig 1 shows an example of a 21/3 reuse structure, where we have $M_{c}=7$ and $L_{c}=3$. Subsequently, the frequency collision rate is expressed as $y=\left(M_{c} k\right) /\left(L_{c} N_{c}\right)$, where $k$ is the fractional overlap factor. Note that we have $k=0$ for cells of different colours while $k=1$ for cells of the same colour and shade. Other values of $k$ may be found in [11]

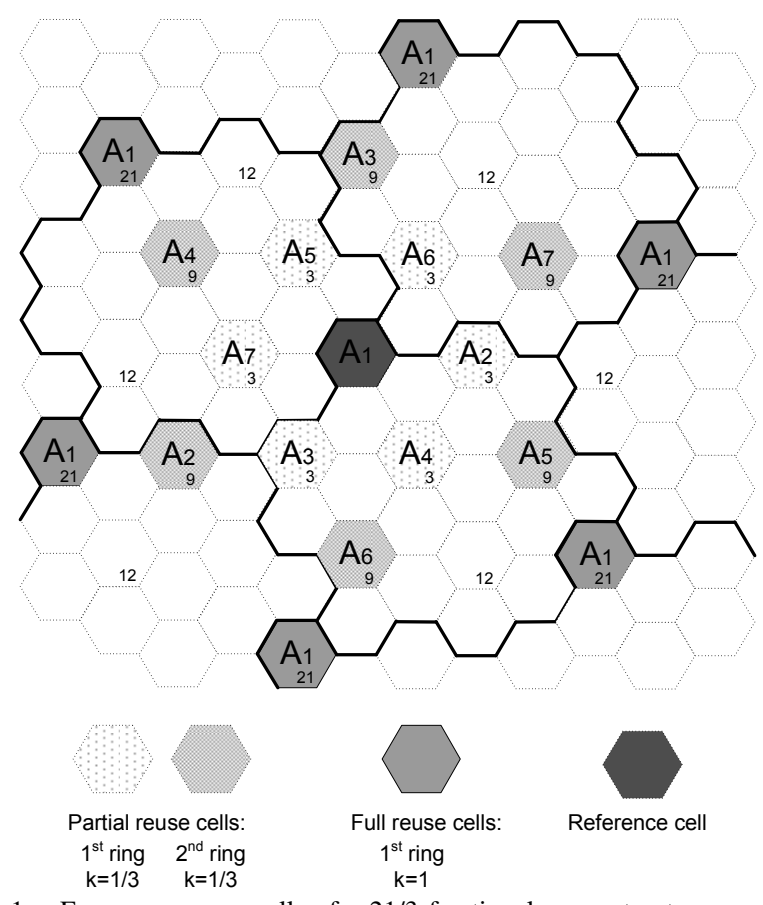

Fig. 1. Frequency reuse cells of a 21/3 fractional reuse structure

\section{Area Spectral EfFiciency of STFSK Aided SFHMA}

\section{A. Area Spectral Efficiency of the SFHMA system}

In this section, the method proposed in [3], [11] will be employed for evaluating the ASE of the proposed STFSK aided SFHMA system. Furthermore, the spectral efficiency of the STFSK aided SFHMA, the GMSK aided SFHMA and the conventional GMSK aided TD/FDMA systems will be compared. In this study we focus our attention on the base-to-mobile DownLink (DL).

In order to calculate the ASE of the entire system, we first consider the probability of correct reception of a hop, $q_{c}$, ensured by the FEC coding and by the audio/video codec of a multimedia session for the sake of guaranteeing an acceptable service quality. According to [3], a $q_{c}$ value of 0.7 is capable of guaranteeing an acceptable speech quality for a system utilizing the Reed-Solomon (RS) $(8,4)$ channel code and a $16 \mathrm{kbit} / \mathrm{s}$ sub-band codec, resulting in an RS-decoded BER of approximately $3 \times 10^{-3}$. It is worth nothing that the stateof-the-art Advanced Multi-Rate (AMR) speech codec [12] provides a good speech quality even at $B E R=10^{-2}$. However, in order to facilitate reliable system acquisition and synchronization, the target BER of $10^{-3}$ is chosen in this paper. The probability $q_{c}$ of correct reception during a hop may be formulated as:

$$
q_{c}=\operatorname{Prob}\{\lambda \geq \gamma\},
$$

where $\gamma$ represents the Energy per Bit to Noise power spectral density ratio $\left(E_{b} / N_{0}\right)$ corresponding to the Bit Error Ratio (BER) threshold and $\lambda$ is the Carrier-to-Interference Ratio (CIR) at the receiver considered, where the interference is imposed by all the adjacent cells. Note that the CIR depends on the received power, on the shadowing and fast-fading parameters, as well as on the interference imposed by other cells in the network. According to [11], Eq. (1) may be expressed as

$$
q_{c}=\prod_{i=1}^{M_{I}}\left(1-\frac{p_{i} \gamma}{\Lambda_{i}+\gamma}\right),
$$

where $M_{I}$ denotes the number of cells imposing interference on the cell considered, while $\Lambda_{i}$ is the CIR corresponding to the interference from the $i^{t h}$ cell. Finally, $p_{i}$ presents the frequencycollision probability.

In line with [11], we assume that the locations of MSs are random in the reference cell. Therefore, $q_{c}$ may be calculated for various values of the tele-traffic load, $X$, which is also known as the average number of users actively engaged in calls per $\mathrm{MHz}$ per cell. In the analysis of [11], the ' $90 \%$ worst case value', $q_{90}$, defined as the specific value of $q_{c}$, which is exceeded with a probability of $90 \%$, is utilized. The study of [3] suggested that the specific operating point where we have $q_{90}=0.7$ in the graph of $q_{90}$ versus $X$ should be selected in order to ensure an acceptable reception quality. However, the analysis of [11] indicated that $q_{90}=0.8$ might be a better choice for the minimum $q$ to determine the ASE. Hence, the value of $q_{90}=$ 0.8 is considered in our following analysis.

The ASE calculation may then be simplified to:

$$
\eta=a_{\text {cell }} / W=X
$$

where $a_{\text {cell }}$ is the traffic load and $W$ is the total allocated bandwidth. Furthermore, the frequency-collision probability may be expressed as

$$
p_{i}=\frac{y_{i} r_{a} W X}{n_{t} n_{\text {call }}}
$$

where $n_{t}$ is the number of slots per TDM frame and $n_{\text {call }}$ is the number of calls per timeslot, while $r_{a}$ is the Voice Activity Ratio (VAR), typically assumed to be 0.5 for the downlink [3]. For a fullreuse cell cluster of size $C_{c}$, the frequency-collision rate $y_{i}$ becomes $C_{c} / 3 N_{c}$. By contrast, we have $y_{i}=k_{i} M_{c} / L_{c} N_{c}$ for the $3 M / L$ fractional reuse structure. As regards to the channel spacing $f_{s}$, the number of hopping frequencies assigned to each of the three basic colours is given by $N=W /\left(3 f_{s}\right)$.

Hence, Eq. (4) may be simplified to

$$
p_{i}=\frac{C_{c} X r_{a} f_{s}}{n_{t} n_{\text {call }}}
$$

for the full-reuse cells and

$$
p_{i}=\frac{3 k_{i} M X r_{a} f_{s}}{L n_{t} n_{\text {call }}}
$$

for fractional reuse.

In reality, most cellular systems employ fixed beam-formingbased angular sectorization, at least near the cell-edge. Thus, in the scenario of $120^{\circ}$ sectorization only 3 of 6 neighbour cells impose interference on the reference cell. However, there are only two cells, which contribute the full interference. Moreover, the authors of [3] demonstrated that the probability of the event, when the worst-case value $q_{90}$ occurs is comparable to the probability of $\Lambda_{90}$, which is defined as the ' $90 \%$ worst-case value' CIR, in other words, $\operatorname{Prob}\left(q_{c}>q_{90}\right)=\operatorname{Prob}\left(\Lambda>\Lambda_{90}\right)=90 \%$. Hence, $q_{90}$ may be expressed as

$$
q_{90}=\prod_{i=1}^{K_{I}}\left(1-\frac{p_{i} \gamma}{2 \Lambda_{i}+\gamma}\right)^{2}
$$


where $K_{I}$ is the number of rings using a different frequency set around the reference cell.

Fig. 2 shows the BER performance of both GMSK and STFSK(4/1/4/2-4-2), where we employ $M / N / T / Q=4 / 1 / 4 / 2$, 4PSK and 2-FSK modulation for transmission over the COST-207 rural area channel model [13], when assuming a maximum multipath delay of $20 \mu \mathrm{s}$, corresponding to an Inter-Symbol Interference (ISI) of 6 symbols at a bit rate of about $300 \mathrm{kbits} / \mathrm{s}$. The half-rate channel codes RS $(8,4)[11]$ and the Recursive Systematic Convolutional (RSC) code $\operatorname{RSC}(23,33)$ using the octally represented generator polynomials of 23 and 33 [14] are considered. For RSC-coded system, soft-decision were used at the receiver. According to Fig. 2, the target BER of $10^{-3}$ was achieved at the $E_{b} / N_{0}$ value of $\gamma=26.0 \mathrm{~dB}, 19.0 \mathrm{~dB}$ and $8.5 \mathrm{~dB}$ for the uncoded, RS coded and RSC coded GMSK schemes, respectively. For the same coding schemes invoked for the STFSK transceiver, the corresponding values are $\gamma=9.5 \mathrm{~dB}, 7.5 \mathrm{~dB}$ and $1.5 \mathrm{~dB}$, respectively. All of these values will be used for computing the system's $q_{90}$ values as well as its ASE.

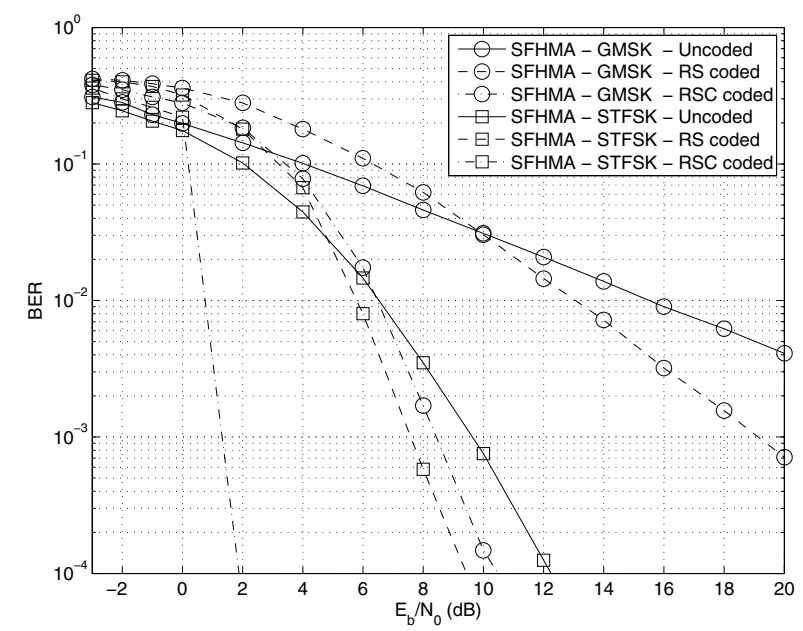

Fig. 2. BER versus $E_{b} / N_{0}$ performance of the GMSK and the STFSK(4/1/4/2-4-2) schemes employing RS and RSC channel codes for transmission over the 6-tap COST-207 rural area channel model subjected to Rayleigh fading and AWGN.

For a basic 3-cell structure using the above values of $\gamma$ as well as $\Lambda_{90}$ from Table 7.4 of [11] and assuming a frequency spacing of $f_{s}=1 / 7 \mathrm{MHz}$, the values of $q_{90}$ versus the average number of users, $X$, are presented in Fig. 3. The curves were plotted for the following conditions:

- Nearest reuse ring (cluster-size 3) only is marked by the circles.

- Nearest reuse ring plus two nearest rings of cells of the other colours which impose adjacent-channel interference, for which the values of $\Lambda_{90}$ taken from [3] are $R_{A C}+2 \mathrm{~dB}$ and $R_{A C}+$ $9 \mathrm{~dB}$, where $R_{A C}=17 \mathrm{~dB}$ is the adjacent-channel interference rejection for $f_{s}=1 / 7 \mathrm{MHz}$. This scenario is indicated by the down-facing triangles.

- The second reuse ring (size 9) only, as indicated by the upwards triangles.

- The first five reuse rings (size $3,9,12,21,27$ ), as marked by the squares.

As seen in the figure, the tiny gaps between the circle-marked curves and the down-facing-marked curves indicate that the adjacent channel interference insignificantly affect the cell's performance. By contrast, due to the co-channel interference, the cell's tele-traffic load $X$ is reduced by approximately 1 users $/ \mathrm{cell} / \mathrm{MHz}$ at $q_{90}=0.8$ when the number of reuse rings increases from one to five. Based on the $q_{90}$ curves, it is possible to find the system's ASE, $\eta_{\text {sys }}$, by spotting the $X$ value, where we have $q_{90}=0.8$. Note that the ASE is quantified in terms of Erlang/cell/MHz, or as $\mathrm{Erl} / \mathrm{cell} / \mathrm{MHz}$ for short.

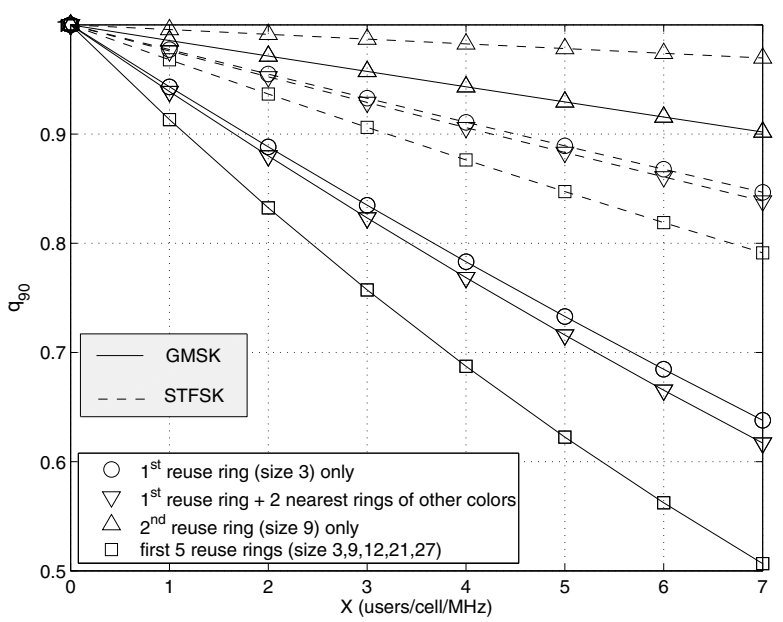

Fig. 3. $q_{90}$ versus $\mathrm{X}$ for GMSK and STFSK(4/1/4/2-4-2) employing RSC channel code for the full-reuse cluster size of 3 and $r_{a}=0.5$

Theoretically, the probability of frequency collision, $p_{i}$, depends on three factors, namely the fractional overlap between the sets of frequencies in the reuse and reference cell, the VAR and the mean channel utilization, $U$. Thus, $p_{i}$ can be expressed as

$$
p_{i}=k_{i} U r_{a} .
$$

Note that $U=1.0$, when all channels are occupied. Using the condition $U=1.0$ and Eqs. (3-4), the maximum ASE may be obtained as

$$
\eta_{\max }=\frac{n_{t} n_{\text {call }}}{C_{c} f_{s}}
$$

for the full-reuse structure and as

$$
\eta_{\max }=\frac{L_{c} n_{t} n_{\text {call }}}{3 M_{c} f_{s}}
$$

for fractional reuse structure.

In practice, the achievable spectral efficiency, $\eta_{a c h}$, of a system, which may be achieved with the aid of the Erlang B formula [15], depends additionally on the number of channels per cell, $n_{c h}$, and on the tolerable blocking probability, $P_{B}$. The achievable ASE may be obtained as

$$
\eta_{\text {ach }}=U \times \eta_{\max }
$$

where $\mathrm{U}$ may be determined by Erlang B equation. Table I shows the maximum as well as the achievable spectral efficiency of various reuse structures, where we have $W=24 \mathrm{MHz}, f_{s}=1 / 7 \mathrm{MHz}$ and $P_{B}=2 \%$.

Consequently, the attainable ASE of a specific system is given by

$$
\eta_{\text {att }}=\min \left\{\eta_{\text {sys }}, \eta_{\text {ach }}\right\} .
$$

The attainable ASE of various reuse structures is provided in Table I. As seen in the table, the STFSK aided SFHMA may triple the ASE compared to the one employing classic GMSK, when no channel coding is employed. When the hard-decision $\operatorname{RS}(8,4)$ channel code was employed, the ASE of the STFSK system is still as twice as high as that of GMSK. Furthermore, with the aid of the soft-decision $\operatorname{RSC}(23,33)$ the STFSK may approach the maximum achievable ASE for all the reuse structures considered. By contrast, this was only possible for the full-reuse cluster size of 9 and for the fractionalreuse cluster size $21 / 3$ in case of the $\operatorname{RSC}(23,33)$ coded GMSK. 
TABLE I

SPECTRAL EFFICIENCY $(\mathrm{Erl} / \mathrm{cell} / \mathrm{MHz})$ FOR VARIOUS REUSE STRUCTURES: $r_{a}=0.5 ; P_{B}=2 \% ; q_{90}=0.8 ; n_{c a l l}=1$

\begin{tabular}{|c|c|c|c|c|c|c|c|c|c|c|}
\hline \multirow{2}{*}{$\begin{array}{l}\text { Cluster } \\
\text { size }\end{array}$} & \multirow[t]{2}{*}{$\eta_{\max }$} & \multirow[t]{2}{*}{$n_{c h}$} & \multirow[t]{2}{*}{$\overline{U_{a c h}}$} & \multirow[t]{2}{*}{$\eta_{\text {ach }}$} & \multicolumn{2}{|c|}{$\eta_{\text {sys }}$} & \multicolumn{2}{|c|}{$\eta_{a t t}$} & \multicolumn{2}{|c|}{$U \%$} \\
\hline & & & & & Uncoded-GMSK & Uncoded-STFSK & Uncoded-GMSK & Uncoded-STFSK & Uncoded-GMSK & Uncoded-STFSK \\
\hline 3 & 7.00 & 168 & 0.901 & 6.31 & 0.44 & 1.55 & 0.44 & 1.55 & 6 & 22 \\
\hline 9 & 2.33 & 56 & 0.803 & 1.87 & 0.39 & 2.28 & 0.39 & 1.87 & 17 & 80 \\
\hline $9 / 2$ & 4.67 & 112 & 0.872 & 4.07 & 0.43 & 1.70 & 0.43 & 1.70 & 9 & 36 \\
\hline $12 / 2$ & 3.50 & 84 & 0.846 & 2.96 & 0.40 & 1.37 & 0.40 & 1.37 & 11 & 39 \\
\hline $12 / 3$ & 5.25 & 126 & 0.881 & 4.63 & 0.44 & 1.64 & 0.44 & 1.64 & 8 & 31 \\
\hline $21 / 3$ & 3.00 & 71 & 0.831 & 2.49 & 0.41 & 1.77 & 0.41 & 1.77 & 14 & 59 \\
\hline $21 / 4$ & 4.00 & 96 & 0.859 & 3.44 & 0.42 & 1.67 & 0.42 & 1.67 & 11 & 42 \\
\hline & & & & & RS-GMSK & RS-STFSK & RS-GMSK & RS-STFSK & RS-GMSK & RS-STFSK \\
\hline 3 & 7.00 & 168 & 0.901 & 6.31 & 0.59 & 2.07 & 0.59 & 2.07 & 8 & 30 \\
\hline 9 & 2.33 & 56 & 0.803 & 1.87 & 0.58 & 3.40 & 0.58 & 2.33 & 25 & 80 \\
\hline $9 / 2$ & 4.67 & 112 & 0.872 & 4.07 & 0.60 & 2.31 & 0.60 & 2.31 & 13 & 49 \\
\hline $12 / 2$ & 3.50 & 84 & 0.846 & 2.96 & 0.51 & 1.85 & 0.51 & 1.85 & 15 & 53 \\
\hline $12 / 3$ & 5.25 & 126 & 0.881 & 4.63 & 0.60 & 2.20 & 0.60 & 2.20 & 11 & 42 \\
\hline $21 / 3$ & 3.00 & 71 & 0.831 & 2.49 & 0.60 & 2.41 & 0.60 & 2.41 & 20 & 80 \\
\hline $21 / 4$ & 4.00 & 96 & 0.859 & 3.44 & 0.60 & 2.25 & 0.60 & 2.25 & 15 & 56 \\
\hline & & & & & RSC-GMSK & RSC-STFSK & RSC-GMSK & RSC-STFSK & RSC-GMSK & RSC-STFSK \\
\hline 3 & 7.00 & 168 & 0.901 & 6.31 & 1.78 & 6.04 & 1.78 & 6.04 & 25 & 86 \\
\hline 9 & 2.33 & 56 & 0.803 & 1.87 & 2.77 & $>7.0$ & 1.87 & 1.87 & 80 & 80 \\
\hline $9 / 2$ & 4.67 & 112 & 0.872 & 4.07 & 1.97 & 6.98 & 1.97 & 4.07 & 42 & 87 \\
\hline $12 / 2$ & 3.50 & 84 & 0.846 & 2.96 & 1.59 & 5.53 & 1.59 & 2.96 & 45 & 85 \\
\hline $12 / 3$ & 5.25 & 126 & 0.881 & 4.63 & 1.88 & 6.50 & 1.88 & 4.63 & 36 & 88 \\
\hline $21 / 3$ & 3.00 & 71 & 0.831 & 2.49 & 2.05 & $>7.0$ & 2.05 & 2.49 & 68 & 83 \\
\hline $21 / 4$ & 4.00 & 96 & 0.859 & 3.44 & 1.92 & 6.64 & 1.92 & 3.44 & 48 & 86 \\
\hline
\end{tabular}

In summary, the attainable ASE can be estimated by the following steps:

- Step 1: Determine the $E_{b} / N_{0}$ threshold, $\gamma$, from Fig. 2 which offers the target BER.

- Step 2: Compute the frequency collision probability, $p_{i}$, from Eq. (5) or Eq. (6).

- Step 3: Calculate q9o from Eq. (7).

- Step 4: Determine the system's ASE, $\eta_{\text {sys }}$ by looking for the operating point in Fig. 3, where we have the target $q_{90}$, i.e $q_{90}=0.8$ in the graph of $q_{90}$ versus $X$.

- Step 5: Calculate the achievable spectral efficiency, $\eta_{\text {ach }}$, from Eq. (11).

- Step 6: Determine the attainable ASE, $\eta_{\text {att }}$, which is the lower of the pair $\left(\eta_{s y s}, \eta_{a c h}\right)$.

\section{B. Comparison between the SFHMA and TD/FDMA systems}

Finally, we compare the attainable performance of STFSK aided SFHMA to that of conventional GMSK aided TD/FDMA, to that of the third-generation WCDMA system, as well as to the performance of the fourth-generation LTE system. The system parameters considered are provided in Table II. In order to support a voice channel employing the AMR speech codec operated for example at $12.2 \mathrm{kbps}$, in the $5 \mathrm{MHz}$-bandwidth WCDMA system a maximum of 98 users can be separated when using a spreading factor of 128 [16]. For the same bandwidth of $5 \mathrm{MHz}$, the LTE system is capable of supporting up to 200 users [17]. Naturally, both the WCDMA and the LTE as well as the STFSK system are capable of supporting a significantly higher data rate than the $12.2 \mathrm{kbps}$ speech rate. However, in this investigation we focussed our attention on the telephony service. Therefore, the voice data rate of $12.2 \mathrm{kbps}$ is selected for all systems considered. As a benefit of employing CDMA in WCDMA and OFDM in LTE, both systems are capable of operating at full frequency reuse in all the adjacent cells.

The performance of the three systems recorded at an $E_{b} / N_{0}$ of $30 \mathrm{~dB}$ is shown in Fig. 4 in terms of the BER versus the mean CIR.
Based on Fig. 4, the threshold BER of $10^{-3}$ is achieved at the mean CIR of $\Lambda_{t h}=12 \mathrm{~dB}, 6.5 \mathrm{~dB}$ and $-1.0 \mathrm{~dB}$ for GMSK-aided TD/FDMA, for GSMK-assisted SFHMA and TSFSK-aided SFHMA, respectively. These values will be used for determining the minimum reuse cluster size. More particularly, the selected reuse cluster size's $\Lambda_{90}$ must be higher than the threshold mean CIR $\Lambda_{t h}$. In other words, the interference power imposed by frequency-reuse cells on the reference cell must be lower than the critical threshold interference level, which may be calculated from the threshold mean CIR $\Lambda_{t h}$. For instance, in order to satisfy the condition of $\Lambda_{90}>\left(\Lambda_{t h}=10.0 \mathrm{~dB}\right)$, the TD/FDMA's smallest reuse cluster size must be the full-reuse 9-cell cluster, where we have $\Lambda_{90}=13.0 d B$ based on Table 7.4 of [11]. Similarly, observe from this table that the minimum cluster size is 3 for both the GMSK and the STFSK aided SFHMA system.

Applying the 6-step process outlined above, the various systems' ASEs are shown in the last line of Table II. According to the table, the GMSK aided TD/FDMA system does not perform as well as the GMSK aided SFHMA arrangement in terms of the ASE, which is in contrast to the results of Chapter 7 in [11]. The reason for this fact is that when strong channel codes, such as the RSC or turbo codes, are employed, the reference BER value can be reduced, resulting in smaller reuse cluster sizes. The advantage of the small reuse cluster sizes in the SFHMA system becomes less dominant. Moreover, owing to the employment of SFH, the SFHMA system requires a longer time slot for each user. Hence, the GMSK aided SFHMA system performs less efficiently than GMSK aided TD/FDMA. However, the cell's performance may be significantly improved by employing STFSK. Quantitatively, STFSK aided SFHMA attained a spectral efficiency of $24.7 \mathrm{Erl} / \mathrm{cell} / \mathrm{MHz}$, outperforming the ASE of 15.0 $\mathrm{Erl} / \mathrm{cell} / \mathrm{MHz}$ recorded for the GMSK aided TD/FDMA system. This ASE is also significantly higher than the WCDMA's ASE of 16.9 $\mathrm{Erl} / \mathrm{cell} / \mathrm{MHz}$, which is only slightly better than that of the GMSK aided TD/FDMA system. Finally, the LTE system achieves an ASE of $36.5 \mathrm{Erl} / \mathrm{cell} / \mathrm{MHz}$, hence exhibiting a substantially better ASE than all the remaining systems considered. 
TABLE II

SPECTRAL EFFICIENCY $(\mathrm{Erl} / \mathrm{cell} / \mathrm{MHz})$ FOR VARIOUS SYSTEMS: $E_{b} / N_{0}=30 d B ; P_{B}=2 \%$

\begin{tabular}{|c|c|c|c|c|c|c|}
\hline \multirow[t]{2}{*}{ System parameters } & & \multirow{2}{*}{$\begin{array}{r}\text { TD-FDMA } \\
\text { GMSK }\end{array}$} & \multicolumn{2}{|c|}{ SFHMA } & \multirow[t]{2}{*}{ WCDMA } & \multirow[t]{2}{*}{ LTE } \\
\hline & & & GMSK & STFSK & & \\
\hline Speech codec & & $\begin{array}{r}\text { AMR } \\
(12.2 \mathrm{kbps})\end{array}$ & $\begin{array}{r}\text { AMR } \\
(12.2 \mathrm{kbps})\end{array}$ & $\begin{array}{r}\text { AMR } \\
(12.2 \mathrm{kbps})\end{array}$ & $\begin{array}{r}\text { AMR } \\
(12.2 \mathrm{kbps})\end{array}$ & $\begin{array}{r}\text { AMR } \\
(12.2 \mathrm{kbps})\end{array}$ \\
\hline Channel code type & & $\operatorname{RSC}(23,33)$ & $\operatorname{RSC}(23,33)$ & $\operatorname{RSC}(23,33)$ & $\operatorname{RSC}(23,33)$ & $\operatorname{RSC}(23,33)$ \\
\hline Modulation type & & GMSK & GMSK & $\begin{array}{r}\text { STFSK } \\
(4 / 1 / 4 / 2-4-2)\end{array}$ & QPSK & QPSK \\
\hline Antenna configuration $(\mathrm{Tx} / \mathrm{Rx})$ & & $1 / 1$ & $1 / 1$ & $4 / 1$ & $1 / 1$ & $4 / 1$ \\
\hline System bandwidth $(\mathrm{MHz})$ & $W$ & 5 & 5 & 5 & 5 & 5 \\
\hline Cluster size & $C$ & 9 & 9 & 3 & 1 & 1 \\
\hline No. of timeslots & $n_{t}$ & 8 & 3 & 3 & 1 & 1 \\
\hline No. of call per timeslot & $n_{\text {call }}$ & 4 & 4 & 4 & 1 & 1 \\
\hline Frequency spacing (MHz) & $f_{s}$ & 0.200 & 0.143 & 0.143 & 5 & 5 \\
\hline No. of frequencies/system & $n_{f_{s}}$ & 25 & 35 & 168 & 1 & 1 \\
\hline Spreading factor & & 1 & 1 & 1 & 128 & 512 \\
\hline No. of channels/cell & $n_{c h}$ & 89 & 47 & 140 & 98 & 200 \\
\hline Traffic/channel $(e r l)$ & $a_{c h}$ & 0.841 & 0.768 & 0.883 & 0.860 & 0.912 \\
\hline Traffic/cell $(e r l)$ & $a_{\text {cell }}$ & 74.85 & 35.80 & 123.48 & 84.31 & 182.43 \\
\hline Spectral efficiency $(\mathrm{Erl} / \mathrm{cell} / \mathrm{MHz})$ & $\eta_{\text {att }}$ & 14.958 & 7.161 & 24.697 & 16.86 & 36.487 \\
\hline
\end{tabular}

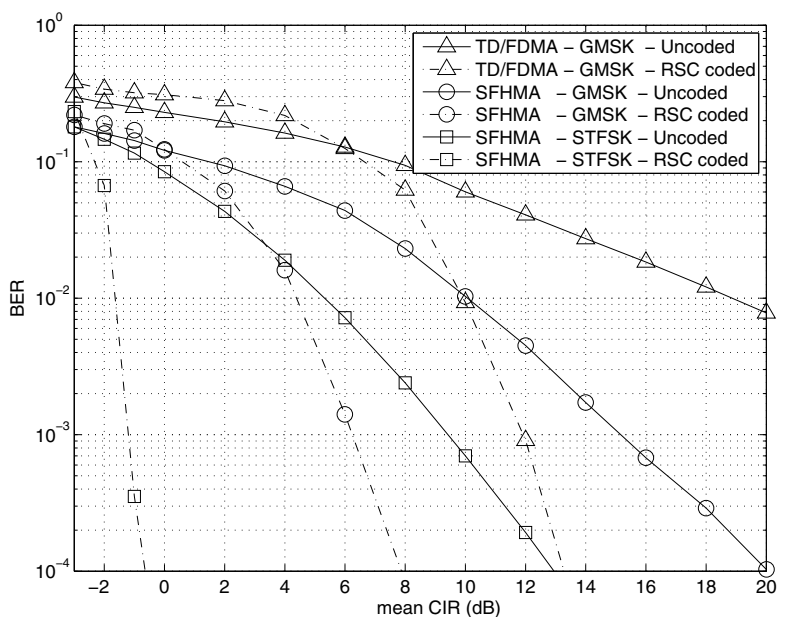

Fig. 4. BER versus mean CIR for GMSK aided TD/FDMA, GMSK aided SFHMA and STFSK(4/1/4/2-4-2) aided SFHMA systems where RSC channel codes are employed in a Rayleigh fading AWGN channel at $E_{b} / N_{0}=30 d B$.

\section{CONCLUSIONS}

In this paper we investigated the ASE of the proposed STFSK aided SFHMA system in various frequency reuse structures. The results showed that the proposed system may double the attainable ASE compared to GMSK aided SFHMA, when the RS $(8,4)$ channel codes are employed for transmission over the 6-tap COST-207 rural area channel model associated with Rayleigh fading and AWGN. Additionally, the soft-decision RSC $(23,33)$ coded STFSK aided SFHMA may approach the maximum achievable ASE in various frequency reuse cluster sizes. By contrast, this is only possible for the fullreuse cluster size 9 and for the fractional-reuse cluster size 21/3 in case of the soft-decision RSC $(23,33)$ coded STFSK aided SFHMA. Furthermore, the system's ASE was compared to that of the softdecision RSC coded GMSK aided TD/FDMA. We demonstrated that the soft-decision RSC $(23,33)$ coded STFSK aided SFHMA system is capable of outperforming the ASE of the RSC $(23,33)$ coded GMSK aided TD/FDMA as well as that of WCDMA. Despite this significant improvement, the ASE of the STFSK aided SFHMA remains only $60 \%$ of that of the more complex LTE system, when the same $\operatorname{RSC}(23,33)$ channel code and a system bandwidth of $5 \mathrm{MHz}$ are employed. Hence in our future research we will find appropriate upper-layer techniques for STFSK in the interest of increasing its ASE.

\section{REFERENCES}

[1] E. Geraniotis and M. Pursley, "Error probabilities for slow-frequencyhopped spread-spectrum multiple-access communications over fading channels," IEEE Transactions on Communications, vol. 30, no. 5, pp. 996-1009, 1982.

[2] D. Verhulst, "Spectrum efficiency analysis of the digital system SFH900," in Proceedings of the 2nd Nordic Seminar Digital Land Mobile, Stockholm, Sweden, 14-16, Oct., 1986.

[3] J.-L. Dornstetter and D. Verhulst, "Cellular efficiency with slow frequency hopping: Analysis of the digital SFH900 mobile system," IEEE Journal on Selected Areas in Communications, vol. 5, no. 5, pp. 835848, 1987.

[4] G. J. Foschini, "Layered space-time architecture for wireless communication in a fading environment when using multi-element antennas," Bell Laboratories Technical Journal, vol. 1, pp. 41-59, 1996.

[5] P. W. Wolniansky, G. J. Foschini, G. D. Golden, and R. A. Valenzuela, "V-BLAST: an architecture for realizing very high data rates over the rich-scattering wireless channel," in Proceedings of URSI International Symposyum on Signals, Systems, and Electronics - ISSSE'98, pp. 295$300,1998$.

[6] S. M. Alamouti, "A simple transmit diversity technique for wireless communications," IEEE Journal on Selected Areas in Communications, vol. 16, no. 8, pp. 1451-1458, 1998.

[7] V. Tarokh, N. Seshadri, and A. R. Calderbank, "Space-time codes for high data rate wireless communication: Performance criterion and code construction," IEEE Transaction on Information Theory, vol. 44, pp. 744-765, 1998.

[8] R. Y. Mesleh, H. Haas, S. Sinanovic, C. W. Ahn, and S. Yun, "Spatial modulation," IEEE Transactions on Vehicular Technology, vol. 57, no. 4, pp. 2228-2241, 2008.

[9] J. Jeganathan, A. Ghrayeb, L. Szczecinski, and A. Ceron, "Space shift keying modulation for MIMO channels," IEEE Transactions on Wireless Communications, vol. 8, no. 7, pp. 3692-3703, 2009.

[10] H. A. Ngo, C. Xu, S. Sugiura, and L. Hanzo, "Space-time-frequency shift keying for dispersive channels," IEEE Signal Processing Letters, vol. 18, no. 3, pp. 177-180, 2011.

[11] R. Steele and L. Hanzo, Mobile Radio Communications: Second and Third-generation Cellular and WATM Systems. John Wiley - IEEE Press, 2nd ed., May 1999.

[12] L. Hanzo, C. Somerville, and J. Woodard, Voice and Audio Compression for Wireless Communications. Wiley-IEEE Press, 2nd ed., Oct. 2007.

[13] M. Patzold, Mobile Fading Channels: Modelling, Analysis and Simulation. New York, NY, USA: John Wiley \& Sons, Inc., 2001.

[14] European Telecommunications Standards Institute, Digital cellular telecommunications system (Phase 2+): Channel coding, 8.5.1 ed., 1999.

[15] International Telecommunication Union - ITU, Teletraffic Engineering Handbook, ITU-D SG 2/16 \& ITC ed., June 2001.

[16] "3GPP Specifications - Series 25: Radio aspects of 3G, including UMTS,” 2001.

[17] "3GPP Specifications - Series 36: LTE (Evolved UTRA) and LTEAdvanced radio technolgy - Release 8," 2007. 